\title{
SÍNDROME DE BECKWITH-WIEDMANN: RELATO DE CASO DA INTERVENÇÃO FONOAUDIOLÓGICA
}

\author{
Beckwith-Wiedemann syndrome: \\ case report of speech therapy intervention
}

\author{
Bárbara de Lavra-Pinto ${ }^{(1)}$, Maria Júlia Luz ${ }^{(2)}$, Ligia Motta ${ }^{(3)}$, Erissandra Gomes ${ }^{(4)}$
}

\begin{abstract}
RESUMO
Tema: descrever a evolução de um caso de Síndrome de Beckwith-Wiedmann com macroglossia submetido à fonoterapia quanto aos aspectos estruturais e miofuncionais do sistema estomatognático. Procedimentos: inicialmente foram coletados os dados do prontuário e foi realizada a entrevista inicial com a mãe. Após, a paciente, com 1 ano e 2 meses de idade, foi submetida à avaliação fonoaudiológica clínica e foram realizados exames complementares (avaliação otorrinolaringológica, exame videofluoroscópico e avaliação audiológica). Após seis meses de terapia miofuncional com a utilização de técnicas passivas, foi realizada uma reavaliação. Resultados: na avaliação fonoaudiológica inicial observou-se flacidez de lábios e bochechas; ausência de vedamento labial; língua com tensão diminuída, hemi-hipertrofia direita e acentuada projeção anterior; dificuldades na mastigação e na deglutição de alimentos sólidos. Decorridos seis meses de terapia miofuncional, constatou-se maior rigidez da musculatura de lábios e bochechas, posteriorização da língua com aumento da tensão muscular, e desempenho adequado na mastigação e deglutição de alimentos sólidos. Conclusão: a intervenção fonoaudiológica precoce, baseada em técnicas de terapia miofuncional, apresentou resultados considerados satisfatórios para este caso de Síndrome de Beckwith-Wiedmann com macroglossia.
\end{abstract}

DESCRITORES: Síndrome de Beckwith-Wiedmann; Macroglossia; Terapia Miofuncional

\section{INTRODUÇÃO}

A Síndrome de Beckwith-Wiedmann foi descrita em 1963 por Beckwith e, em 1964, por Wiedmann, o que originou o nome da síndrome ${ }^{1}$. A mesma é

(1) Fonoaudióloga; Universidade Federal do Rio Grande do Sul, UFRGS, Porto Alegre, RS, Brasil; Especialista em Motricidade Orofacial pelo CEFAC - Pós-Graduação em Saúde e Educação; Mestre em Linguística pela Pontifícia Universidade Católica do Rio Grande do Sul.

(2) Fonoaudióloga; Clínica Vocare, São Leopoldo, RS, Brasil; Especialista em Motricidade Orofacial pelo CEFAC - PósGraduação em Saúde e Educação.

(3) Fonoaudióloga; Hospital São Lucas da Pontifícia Universidade Católica do Rio Grande do Sul, PUCRS, Porto Alegre, RS, Brasil; Mestre e Doutoranda em Gerontologia Biomédica na Pontifícia Universidade Católica do Rio Grande do Sul.

(4) Fonoaudióloga; Docente do curso de Fonoaudiologia da Universidade Federal de do Rio Grande do Sul, UFRGS, Porto Alegre, RS, Brasil; Doutora em Ciências Médicas: Pediatria pela Universidade Federal do Rio Grande do Sul.

Conflito de interesses: inexistente causada por uma alteração no braço curto do cromossomo 11, mas o processo responsável pelos sinais clínicos permanece dúbio ${ }^{2}$.

Tal patologia se manifesta esporadicamente (85\% dos casos) ou como resultado de transmissão hereditária ${ }^{2,3}$. Herdeiros do gene materno sofrem completamente da síndrome e os de gene paterno mostram apenas sinais leves ${ }^{2}$. Sua incidência é de 1:17000 nascidos ${ }^{1,3,4}$, com maior prevalência no sexo feminino ${ }^{5}$. Contudo, essa proporção é subestimada, pois muitas crianças diagnosticadas e com óbito precoce, assim como pacientes menos afetados não têm sido classificados. O diagnóstico da síndrome é feito por sinais clínicos e pode ser confirmada por exame genético molecular ${ }^{6}$. Estudos atuais mostram a possibilidade de diagnóstico pré-natal ${ }^{7,8}$.

Dentre a variedade de sinais clínicos, pode-se identificar uma tríade distinta de anomalias congênitas: onfalocele (hérnia umbilical congênita), gigantismo e macroglossia ${ }^{2,4,5}$. É possível, ainda, encontrar: hipoglicemia neonatal, leve microcefalia, 
hemangioma capilar na fronte, sulcos anormais nos lóbulos das orelhas, exoftalmo, dorso nasal alargado com aplainamento das cartilagens alares, proeminência occipital, hipoplasia maxilar, assoalho orbitário raso e hipoplasia do terço médio da face ${ }^{9}$. A probabilidade de desenvolvimento de tumores malignos é alta, os quais normalmente são constatados antes dos dez anos de idade ${ }^{1-3}$.

A macroglossia é o sinal mais frequente ${ }^{4} \mathrm{com}$ índices de ocorrência entre $82 \%$ a $99 \%$ dos casos ${ }^{3}$. Na Síndrome de Beckwith-Wiedmann, a macroglossia é verdadeira e de origem congênita, caracterizada pela presença de anormalidades histológicas, observando-se aumento das estruturas linguais normais (canais vasculares, elementos glandulares e fibras musculares) ou presença de elementos anormais, como tumores e cistos ${ }^{5}$. O diagnóstico pode ser realizado por meio da verificação de sinais clínicos ${ }^{10}$. Os sinais de macroglossia descritos na literatura e encontrados neste estudo de caso são: projeção anterior acentuada da língua com posição habitual permanentemente entre as arcadas dentárias, hipertrofia muscular e dificuldades na mastigação, deglutição e fala. A língua volumosa acarreta alterações nas arcadas dentárias, o que gera desequilíbrio entre os grupos musculares, favorecendo a flacidez da musculatura orofacial (lábios e bochechas) ${ }^{5}$.

O tratamento médico neonatal da síndrome deve preconizar o controle da hipoglicemia e a correção cirúrgica da onfalocele. No período pós-natal, a criança deverá seguir com acompanhamento pediátrico e avaliações complementares deverão ser solicitadas. Dentre elas, destacam-se: avaliação ortopédica, cardiológica, otorrinolaringológica, bucomaxilofacial, fonoaudiológica e ortodôntica.

O tratamento da macroglossia requer avaliação criteriosa da relação entre o tamanho da cavidade oral e da língua (conteúdo/continente), e de suas implicações dento-esquelético-funcionais antes da aplicação de qualquer procedimento clínico ou cirúrgico ${ }^{5}$. Essa avaliação deve incluir exames clínicos, radiográficos e funcionais, com o objetivo de verificar a interferência na respiração, fala, mastigação e deglutição ${ }^{10}$. A ressecção cirúrgica parcial durante primeiros três anos de vida é o tratamento comumente indicado. O objetivo da cirurgia, na maioria dos casos, é prevenir ou minimizar deformidades maxilofaciais e distorções da imagem corporal que possam vir a afetar o convívio social da criança ${ }^{5,11,12}$. Um estudo longitudinal realizado no período pós-cirúrgico relata casos de pacientes que não alcançaram a completa normalidade das funções da língua, além de diminuição da sensibilidade gustativa ${ }^{13}$.
Entrementes, na literatura pesquisada, alguns autores priorizam condutas não invasivas, sugerindo avaliação e parecer da fonoaudiologia quanto à capacitação das funções orofaciais nas crianças com macroglossia ${ }^{2,14,15}$. O tratamento fonoaudiológico pode ser indicado, devendo iniciar precocemente, no primeiro ano de vida, fase de maior plasticidade neuronal. $O$ intuito é facilitar a aquisição e fixação das praxias orofaciais; adequar a postura e o tônus dos órgãos fonoarticulatórios; propiciar um desempenho satisfatório nas funções do sistema sensitivo-motor oral, principalmente a deglutição, a mastigação e a fala ${ }^{5,14}$.

As alterações orofaciais apresentadas pelos pacientes portadores da Síndrome de BeckwithWiedmann requerem uma abordagem multidisciplinar, na qual os tratamentos indicados se complementam, não somente para alcançar um posicionamento correto da língua e dentes na cavidade oral, mas, principalmente, para proporcionar um adequado desempenho de funções fisiológicas e um correto desenvolvimento neuromuscular ${ }^{2}$.

Com base no descrito acima, o objetivo deste estudo foi descrever a evolução de um caso de Síndrome de Beckwith-Wiedmann com macroglossia submetido à fonoterapia quanto aos aspectos estruturais e miofuncionais do sistema estomatognático, considerando as funções de mastigação, deglutição e fala.

\section{APRESENTAÇÃO DO CASO}

Este estudo é um relato de caso do atendimento fonoaudiológico a um paciente do sexo feminino, com diagnóstico clínico da Síndrome de Beckwith-Wiedmann. A paciente foi encaminhada pelo ambulatório de Pediatria para o ambulatório de Fonoaudiologia do Hospital São Lucas da Pontifícia Universidade Católica do Rio Grande do Sul (PUCRS), devido a dificuldades para deglutir alimentos sólidos.

Inicialmente, foram coletados dados do prontuário da paciente, no qual se verificou nascimento com 31 semanas e cinco dias de gestação, com quadro cianótico e peso $1,875 \mathrm{~kg}$. A criança foi transferida para a UTI neonatal na primeira hora de vida. Durante o período de internação, administrouse ampicilina e gentamicina, a criança foi submetida a tratamento para icterícia, apresentou quadros de hipoglicemia e apneia e recebeu alimentação via sonda nasogástrica. No diagnóstico médico foram descritos sinais clínicos compatíveis com a Síndrome de Beckwith-Wiedmann como a macroglossia, hérnia umbilical e hipoglicemia neonatal. A paciente teve alta após dois meses de internação com adequação da hipoglicemia e alimentação 
por via oral. Nessa época, estava sendo oferecido à criança leite na mamadeira. Não houve acompanhamento fonoaudiológico durante o período de internação hospitalar.

A criança continuou em acompanhamento médico-pediátrico ambulatorial e, com seis meses de vida, realizou avaliação com cirurgião bucomaxilofacial, o qual contra-indicou a glossectomia até crescimento ósseo completo. Entretanto, de acordo com essa avaliação, no caso de prejuízo na realização de funções vitais, devido ao tamanho da língua, a contra-indicação deverá ser reavaliada. $\mathrm{Na}$ avaliação com médico ortopedista foram diagnosticadas hipertrofia de hemicorpo à direita e diferença no comprimento dos membros inferiores. Aos 13 meses, a paciente foi submetida à cirurgia de correção da hérnia umbilical.

A intervenção fonoaudiológica iniciou em abril de 2007, quando a paciente estava com um ano e dois meses de idade cronológica, sem correção com a idade gestacional ao nascimento. Na entrevista inicial com a mãe da criança foram coletados dados sobre a sua história alimentar, hábitos orais, aspectos respiratórios, sono, desenvolvimento psicomotor e linguístico. No momento da avaliação inicial, a queixa da mãe era que a criança não ingeria alimentos sólidos, apresentando reflexo de vômito durante a alimentação.

Os procedimentos iniciais de avaliação realizados foram: avaliação miofuncional orofacial, exame clínico da deglutição, avaliação otorrinolaringológica, exame de videofluoroscopia e avaliação audiológica. Para a avaliação das estruturas orofaciais utilizou-se o Protocolo de Avaliação Miofuncional Orofacial ${ }^{16}$ modificado pelas autoras deste artigo. O protocolo utilizado para a avaliação clínica da deglutição foi elaborado pelas autoras com base em um estudo sobre a atuação fonoaudiológica com recém-nascidos e lactentes disfágicos ${ }^{17}$.

O tratamento fonoaudiológico foi baseado em técnicas de terapia miofuncional ${ }^{5,14}$. Priorizou-se a realização de técnicas passivas (massagens intra e extra-orais) a fim de alcançar o aumento da rigidez muscular da língua, dos lábios e das bochechas. Foram realizadas massagens manuais e com a utilização de um material de estimulação intra-oral (escova arredondada). Para a retroposição da língua e aumento da tensão da musculatura intrínseca, a principal técnica utilizada foi uma massagem intra-oral, com deslizamento da escova de forma suave e rápida no terço posterior até o ápice da língua, fazendo-se, nesse local, leve pressionamento para trás, o que provocou a contração das fibras musculares. $\mathrm{O}$ afilamento da língua foi estimulado por meio de deslizamentos póstero-anteriores com o polegar e o dedo indicador nas laterais da língua. O canolamento da língua foi estimulado utilizando-se a escova arredondada. Realizar canolamento de língua é importante para um adequado desempenho durante a fase oral da deglutição ${ }^{18}$.

$O$ aumento da rigidez da musculatura das bochechas foi alcançado por meio da realização de movimentos ascendentes com a escova arredondada na face oral das bochechas. Esses movimentos foram reforçados com massagens extra-orais simultâneas bilaterais ${ }^{5}$. Em função da assimetria entre as bochechas (direita com maior volume), para cada movimento do lado esquerdo realizaram-se dois do lado direito.

Para promover o aumento da rigidez do músculo orbicular dos lábios e diminuir a eversão do lábio inferior, utilizaram-se exercícios de sopro, estalo de lábios retraídos e protruídos, e a técnica de estimulação da zona motora do mento. Para a realização dessa técnica, coloca-se o dedo indicador sob o soalho da boca para mantê-la fechada, procedendo-se o alisamento, a pressão e a vibração do mento com a extremidade do polegar na direção dorso-caudal ${ }^{19}$.

A fala foi estimulada em atividades lúdicas, priorizando-se a produção de palavras com fonemas bilabiais e labiodentais. A articulação de palavras com fonemas bilabiais facilita a propriocepção do contato entre os lábios ${ }^{15}$. Em alguns momentos, quando a paciente realizava vedamento labial, leves pressionamentos manuais eram realizados buscando maior contato entre os lábios.

A mastigação e a deglutição de alimentos sólidos foram estimuladas, inicialmente, com a utilização de um material em forma de chupeta. Esse material tem uma tela no lugar do bico, onde se colocam os alimentos para a criança mastigar. Foram oferecidos à paciente alimentos sólidos diversificados. A mãe recebeu orientações em relação à consistência dos alimentos que deveriam ser oferecidos e quanto à postura adequada para evitar intercorrências durante a deglutição. Um material semelhante foi utilizado por Mühle e Limongi ${ }^{15}$ para estimulação da mastigação de uma criança com Síndrome de Beckwith-Wiedmann.

Após seis meses de fonoterapia com sessões quinzenais, a paciente foi reavaliada com a utilização dos mesmos protocolos de avaliação inicial. Realizou-se, também, nova avaliação otorrinolaringológica.

Este estudo foi aprovado pelo Comitê de Ética em Pesquisa da PUCRS sob protocolo no 07/4001. O Termo de Consentimento Livre e Esclarecido, assinado pela mãe da criança, autoriza a publicação dos resultados do tratamento e de fotografias da paciente em artigos científicos. Os resultados deste estudo são apresentados sob forma 
descritiva, comparando-se os dados das avaliações mencionadas acima e de imagens obtidas no início da fonoterapia, aos três meses e aos seis meses posteriores. Os principais parâmetros de avaliação para a análise da evolução do caso foram: aumento da tensão muscular da língua ${ }^{20}$, da rigidez da musculatura das bochechas e dos lábios, além de modificações na postura habitual da língua. A possibilidade de vedamento labial e uma maior adequação no desempenho das funções orofaciais de deglutição, mastigação e fala também foram parâmetros importantes para a análise dos resultados. Considera-se uma limitação deste estudo o fato de as fotografias da paciente não terem sido obtidas de forma sistemática e padronizada, o que pode interferir na verificação dos resultados.

\section{RESULTADOS}

As Tabelas 1, 2 e 3 mostram os dados das avaliações clínicas fonoaudiológicas realizadas no início do tratamento e após seis meses de terapia miofuncional. Na Tabela 1 constam os resultados da avaliação inicial e final das estruturas orofaciais. Cavidade oral ampla foi um aspecto observado nos dois momentos de avaliação. A Tabela 2 se refere às avaliações inicial e final das funções do sistema estomatognático: sucção, respiração, fala, mastigação e deglutição. A Tabela 3 mostra os resultados dos exames clínicos da deglutição com diferentes consistências de alimentos no início da fonoterapia e após 6 meses de tratamento. O uso do material para estimulação da mastigação propiciou um desempenho satisfatório na mastigação e deglutição de alimentos sólidos em três meses de terapia miofuncional. Após o tratamento fonoaudiológico, a queixa relacionada à deglutição deixou de existir e a criança passou a ingerir alimentos sólidos.

As Figuras 1, 2, 3 ilustram três diferentes momentos da paciente: anterior ao tratamento fonoaudiológico, aos três meses de fonoterapia e no sexto mês de atendimento fonoaudiológico.

Os resultados da oroscopia realizada na primeira avaliação otorrinolaringológica foram: macroglossia com musculatura hipertrófica à direita e tonsilas palatinas entre os graus II - III, o que caracteriza uma hipertrofia moderada ${ }^{21}$. A nasofibroscopia revelou tonsilas faríngeas ocupando $50 \%$ do cavum, epiglote em ômega, pregas aritenoide e epiglótica curtas. A paciente foi encaminhada para avaliação auditiva e realização do exame de vídeofluoroscopia.

$\mathrm{Na}$ avaliação auditiva foram observadas: emissões otoacústicas evocadas transientes e por produto de distorção presentes em ambas orelhas; reações positivas e procura da fonte sonora na avaliação comportamental; medidas de imitância acústica com curvas timpanométricas, complacência estática normais e reflexos acústicos presentes em todas as frequências testadas.

Tabela 1 - Avaliação inicial e final das estruturas orofaciais

\begin{tabular}{|c|c|c|}
\hline & Avaliação inicial & Avaliação após 6 meses de fonoterapia \\
\hline Lábios & $\begin{array}{l}\text { abertos permanentemente; lábio } \\
\text { inferior com eversão, servindo de } \\
\text { apoio para a língua; ambos } \\
\text { flácidos }\end{array}$ & $\begin{array}{l}\text { entre-abertos, porém com possibilidade de } \\
\text { vedamento em alguns momentos; diminuição } \\
\text { da eversão do lábio inferior; aumento da } \\
\text { rigidez muscular labial }\end{array}$ \\
\hline Língua & $\begin{array}{l}\text { assimétrica (hemi-hipertrofia } \\
\text { direita), com projeção anterior } \\
\text { (permanentemente fora da } \\
\text { cavidade oral) e tensão muscular } \\
\text { diminuída }\end{array}$ & $\begin{array}{l}\text { diminuição da assimetria e da projeção } \\
\text { anterior (alternando momentos dentro e fora } \\
\text { da cavidade oral), aumento da tensão } \\
\text { muscular }\end{array}$ \\
\hline Bochechas & $\begin{array}{l}\text { assimétricas (direita com maior } \\
\text { volume) e flácidas }\end{array}$ & $\begin{array}{l}\text { diminuição da assimetria e aumento rigidez } \\
\text { muscular }\end{array}$ \\
\hline Mentual & $\begin{array}{l}\text { rígido por compensação do lábio } \\
\text { inferior com eversão }\end{array}$ & diminuição da rigidez \\
\hline Arcada dentária & ausência de elementos dentários & $\begin{array}{l}\text { presença de } 5 \text { elementos dentários: incisivos } \\
\text { centrais superiores, inferiores e incisivo } \\
\text { lateral esquerdo }\end{array}$ \\
\hline Palato duro & sem alterações & sem alterações \\
\hline Palato mole & sem alterações & sem alterações \\
\hline Tonsilas palatinas & direita mais desenvolvida & direita mais desenvolvida \\
\hline Orelhas & sem alterações & sem alterações \\
\hline Olhos & sem alterações & sem alterações \\
\hline
\end{tabular}


Tabela 2 - Avaliação inicial e final das funções do sistema estomatgnático

\begin{tabular}{|c|c|c|}
\hline & Avaliação inicial & Avaliação após 6 meses de fonoterapia \\
\hline Sucção & $\begin{array}{l}\text { eficiente, porém sem vedamento } \\
\text { labial (língua anteriorizada) }\end{array}$ & com vedamento labial \\
\hline Respiração & oronasal & oronasal \\
\hline Mastigação & $\begin{array}{l}\text { amassamento do alimento com a } \\
\text { língua contra o palato duro e } \\
\text { projeção anterior da língua }\end{array}$ & $\begin{array}{l}\text { corte do alimento com incisivos centrais, } \\
\text { mastigação de alimentos sólidos e, em } \\
\text { alguns momentos, com vedamento labial }\end{array}$ \\
\hline Deglutição & projeção anterior de língua & diminuição da projeção anterior da língua \\
\hline Fala & $\begin{array}{l}\text { vocabulário de expressão: } \\
4 \text { palavras }\end{array}$ & $\begin{array}{l}\text { aumento do vocabulário: em torno de } 20 \\
\text { palavras, incluindo vocábulos com fonemas } \\
\text { bilabiais e labiodentais }\end{array}$ \\
\hline
\end{tabular}

Tabela 3 - Exame clínico inicial e final da deglutição com diferentes consistências

\begin{tabular}{lll}
\hline & Avaliação inicial & Avaliação após 6 meses de fonoterapia \\
\hline Consistências & deglutição com projeção anterior & diminuição da projeção anterior da língua e \\
líquida e pastosa & da língua e escape de alimento; & do escape de alimento durante a deglutição; \\
& ausência de sinais sugestivos de & ausência de sinais de sugestivos de \\
& alteração de deglutição em fase & alteração de deglutição em fase faríngea \\
faríngea (tosse, engasgo, vômito) & (tosse, engasgo, vômito) \\
Consistência & fase oral com amassamento do & fase oral com vedamento labial e língua no \\
sólida & alimento e sem vedamento labial; & interior da cavidade oral em alguns \\
& durante a deglutição observou-se & momentos; durante a deglutição observou-se \\
& projeção anterior da língua, & diminuição da projeção anterior da língua e \\
& reflexo de vômito e irritabilidade; & ausência dos sinais sugestivos de alteração \\
& choro após a alimentação & de deglutição presentes na 1ª avaliação \\
\hline
\end{tabular}

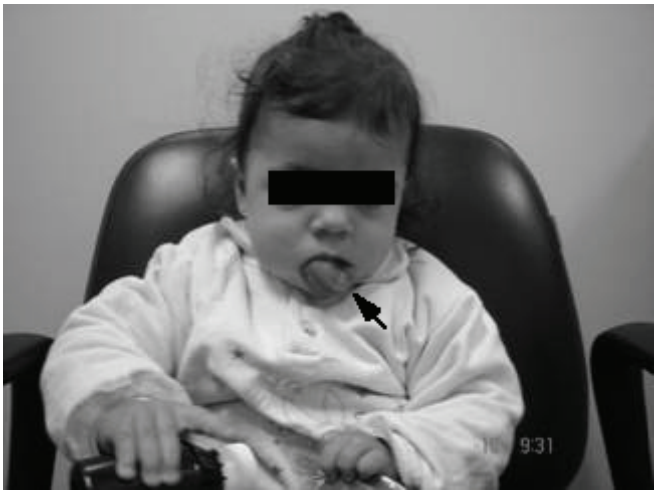

Figura 1 - Anterior ao tratamento fonoaudiológico: língua com tensão muscular diminuída, acentuada projeção anterior e hemi-hipertrofia direita (assimetria)

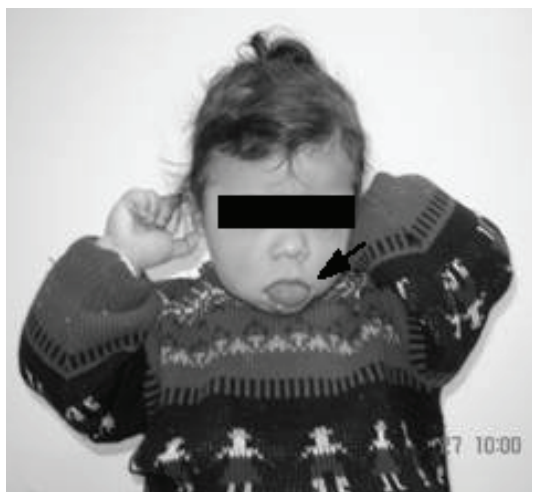

Figura 2 - Aos três meses de fonoterapia: diminuição da projeção anterior da língua e da assimetria

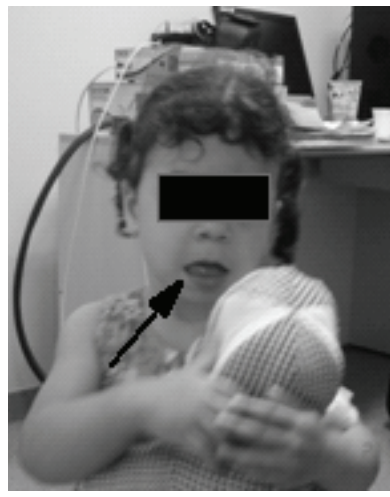

Figura 3 - Aos seis meses de fonoterapia: possibilidade de posicionamento da língua no interior da cavidade oral 
Para a realização do exame de vídeofluoroscopia foram administrados alimentos nas consistências sólido, líquido e pastoso. O parecer funcional indicou inadequação na fase oral da deglutição. Foram observados: escape extra-oral do alimento; movimento de língua adaptado para preparação, organização e ejeção do alimento na cavidade oral (língua projetada para fora da boca durante a preparação do alimento); escape precoce do alimento para a região faríngea com imediata deglutição; ausência de estase de alimento em região faríngea, de aspiração traqueal e de penetração laríngea.

$\mathrm{Na}$ avaliação otorrinolaringológica, após seis meses de fonoterapia, foi sugerido continuidade do tratamento fonoaudiológico pelos resultados satisfatórios alcançados. Não foi possível realizar novo exame de nasofibroscopia devido a problemas técnicos no aparelho. A reavaliação clínica da deglutição não indicou necessidade de realização de um novo exame objetivo da deglutição (vídeofluoroscopia).

Considerou-se que o caso de Síndrome de Beckwith-Wiedmann descrito teve uma boa evolução com seis meses de terapia miofuncional no que se refere aos aspectos estruturais e miofuncionais do sistema estomatognático.

\section{DISCUSSÃO}

No presente estudo de caso, considerando o prognóstico da Síndrome de Beckwith-Wiedmann em relação ao crescimento somático global (macrossomia), a idade da paciente, e as avaliações realizadas (exame miofuncional, bucomaxilofacial, otorrinolaringológico e vídeofluoroscopia), nas quais se constatou que o tamanho da língua não estava impedindo a realização de funções vitais, a opção para o tratamento da macroglossia foi a terapia miofuncional. Também foi levado em consideração o fato da paciente apresentar cavidade oral ampla e a possibilidade de, completado o desenvolvimento ósseo, haver maiores condições para a acomodação da língua ${ }^{13}$. Em grande parte dos estudos sobre macroglossia, o tratamento indicado é a glossectomia parcial 6,10-13. Alguns autores relatam que, após a cirurgia, podem ocorrer alterações relacionadas à percepção de gosto e à articulação de sons da fala ${ }^{6,13}$. Outros, no entanto, ressaltam que a sensibilidade gustativa normalmente não é afetada com a cirurgia e que a fala e a mordida aberta anterior poderão corrigir-se espontaneamente ${ }^{22}$. Outras complicações pós-cirúrgicas encontradas na literatura são: anquilose, língua globular e insensibilidade da ponta da língua ${ }^{11}$.

Apesar de a glossectomia parcial ainda ser 0 tratamento mais frequentemente indicado, a terapia miofuncional como conduta inicial no tratamento da macroglossia nos primeiros anos de vida tem sido sugerida por alguns autores 1,2,5,14,15. Na literatura especializada existe um número reduzido de referências a condutas menos invasivas como procedimento alternativo à glossectomia parcial. Giancotti et al. ${ }^{2}$ relataram um caso de Síndrome de BeckwithWiedmann com macroglossia em que o uso de aparelhos ortodônticos foi o único tratamento utilizado. Dios et al. ${ }^{1}$ descreveram o caso de uma criança com a mesma síndrome, no qual foi indicada a mioterapia baseada na técnica de Castillo-Morales como procedimento inicial. Mühle e Limongi ${ }^{15}$ elaboraram uma proposta de intervenção fonoaudiológica precoce para uma criança de nove meses de idade com Síndrome Beckwith-Wiedmann e macroglossia. Picinato et al. ${ }^{23}$ utilizaram a terapia miofuncinal como tratamento para uma criança de três anos portadora de macroglossia. As autoras citadas relataram que a terapia miofuncional precoce mostrou-se significativa sob todos os aspectos avaliados nos casos descritos. D'Agostino et al. ${ }^{14}$ descreveram um caso de Síndrome de BeckwithWiedmann com acompanhamento fonoaudiológico sequencial por 16 anos, sem a necessidade de intervenções cirúrgicas. O tratamento utilizado nesse caso foi a terapia miofuncional associada ao uso de um aparelho de contenção da língua. Com essa abordagem, obteve-se uma retroposição da língua coaptada ao palato, fechamento gradativo da mordida aberta anterior na segunda dentição e vedamento labial. A finalização desse caso foi descrita por D'Agostino e Costa ${ }^{5}$.

O plano de tratamento fonoaudiológico do caso que está sendo descrito foi elaborado com base no planejamento terapêutico relatado pelas autoras citadas acima ${ }^{5}$. Sugere-se que, nos primeiros três anos, sejam executados exercícios isométricos para aumento da tensão da musculatura da língua, do tônus do músculo orbicular dos lábios e da musculatura das bochechas, principalmente bucinadores.

Atingidos os objetivos da primeira fase, inicia-se a segunda etapa do tratamento, a qual ocorre concomitante ao uso do aparelho para contenção da língua. A terapia miofuncional deve obedecer, de acordo com as autoras, a sequência tônus / postura / função ${ }^{5}$. O encaminhamento para avaliação ortodôntica cefalométrica será importante para o acompanhamento do crescimento ósseo facial. Futuramente, desarmonias oclusais poderão ser corrigidas mediante tratamento ortodôntico ou ortocirúrgico. No presente estudo de caso, para promover o aumento da rigidez da muculatura da língua, dos lábios e das bochechas foram utilizadas técnicas passivas de terapia miofuncional (massagens intra e extra-orais) já descritas na apresentação do caso. 
O exercício da mastigação e a melhora gradativa na postura da língua contribuíram para o início da erupção dentária da paciente. A postura habitual da língua pode influenciar o crescimento e o desenvolvimento de estruturas orofaciais ${ }^{24}$. O aumento da tensão da musculatura da língua e da rigidez muscular dos lábios e das bochechas da criança tornou a postura dessas estruturas mais adequada, ocasionando, assim, um melhor desempenho na realização das funções de mastigação e deglutição. Dagostino e Costa ${ }^{5}$ relataram resultados semelhantes durante a primeira fase de terapia miofuncional em casos de Síndrome de Beckwith-Wiedmann.

\section{CONCLUSÃO}

Após seis meses de tratamento fonoaudiológico, foram alcançados resultados satisfatórios quanto aos aspectos estruturais e miofuncionais do sistema estomatognático. O equilíbrio muscular orofacial que foi estabilizado possibilitou desempenho com maior eficácia na realização das funções de mastigação, deglutição e fala. A intervenção fonoaudiológica, fundamentada em técnicas de terapia miofuncional, foi eficaz como conduta inicial no tratamento da macroglossia para o caso de Síndrome de Beckwith-Wiedmann descrito. Após avaliação multidisciplinar, a terapia miofuncional foi uma alternativa menos invasiva que a glossectomia parcial no tratamento da macroglossia para este caso de Síndrome de Beckwith-Wiedmann. Para que a estabilização dos resultados seja alcançada, atente-se à continuidade do tratamento fonoaudiológico, assim como ao encaminhamento para avaliação ortodôntica. Um estudo ortodôntico cefalométrico deverá ser realizado, haja vista que a macroglossia pode gerar desarmonias no desenvolvimento ósseo facial.

\section{AGRADECIMENTOS}

À fonoaudióloga Lídia D’Agostino pela importante orientação para a realização das técnicas de terapia miofuncional utilizadas neste estudo.

\begin{abstract}
Background: to describe structural and myofunctional aspects of the stomatognathic system for a case of Beckwith-Wiedmann syndrome with macroglossia that received speech therapy intervention. Procedures: first, the patient clinical data was collected from hospital files. An initial interview was carried through with the mother. Then, the patient was submitted to a clinical myofunctional evaluation. Some additional complementary examinations were also held (otolaryngological assessment, videofluoroscopy examination and audiological evaluation). After six months of myofunctional therapy using passive techniques, the patient was revaluated. Results: in the first myofunctional evaluation we noted lips and cheeks' flabbiness, absence of lip seal and tongue with reduced tension, right hemihypertrophy and significant anterior projection. Difficulties in mastication and swallowing of solid food were also observed. After six months of myofunctional therapy, an increase in lips and cheeks' muscle stiffness, tongue posteriorisation with superior muscular tension, and appropriate performance in mastication and swallowing of solid food were evidenced. Conclusion: a precocious speech-therapy intervention, based on myofunctional therapy techniques, showed good results in this case - Beckwith-Wiedmann syndrome with macroglossia.
\end{abstract}

KEYWORDS: Beckwith-Wiedmann Syndrome; Macroglossia; Myofunctional Therapy

\section{REFERÊNCIAS}

1. Dios PD, Posse JL, Sanromán JF, García EV. Treatment of macroglossia in a child with BeckwithWiedemann syndrome. J Oral Maxillofac Surg. 2000 Sep; 58(9):1058-61.

2. Giancotti A, Romanini G, Di Girolamo R, Arcuri C. A less-invasive approach with orthodontic treatment in Beckwith-Wiedemann patients. Orthod Craniofac Res. 2002; 5(1):59-63.

3. Engström W, Lindham S, Schofield $P$. Wiedemann-Beckwith syndrome. Eur J Pediatr. 1988; 147(5):450-7.

4. Clauser L, Tieghi R., Polito J. Treatment of macroglossia in Beckwith-Wiedemann syndrome. J Craniofac Surg. 2006; 17(2):369-72. 
5. D'Agostino L, Costa CSSA. Síndrome de Beckwith-Wiedmann. In: Rocha LD, Zanini SA, Thomé R, Mélega JM, organizadores. Cirurgia plástica, fundamentos e arte: cirurgia reparadora de cabeça e pescoço. Rio de Janeiro: Medsi; 2002. p.530-8.

6. Matamala GN, Toro MAF, Ugarte EV, Mendonza ML. Beckwith Wiedemann syndrome: presentation of a case report. Med Oral Patol Cir Bucal. 2008; 13(10):E640-3.

7. Williams DH, Gauthier DW, Maizels M. Prenatal diagnosis of Beckwith-Wiedemann syndrome. Prenat Diagn. 2005; 25(10):879-84 .

8. Aagaard-Tillery KM, Buchbinder A, Boente MP, Ramin KD. Beckwith-Wiedemann syndrome presenting with an elevated triple screen in the second trimester of pregnancy. Fetal Diagn Ther. 2007; 22(1):18-22.

9. Chen CP. Syndromes and disorders associated with omphalocele (I): Beckwith-Wiedemann syndrome. Taiwan J Obstet Gynecol. 2007; 46(2):96-102.

10. Martinez LP. Macroglossia: etiología multifactorial, manejo múltiple. Columb Méd. 2006; 37(1):67-73.

11. Zerati F, Pirana S, Queiroz E, Sennes LU. Ressecção parcial de língua em paciente com macroglossia portador de síndrome de Beckwith-Wiedemann: relato de caso. Rev Bras Otorrinolaringol. 2005; 71(3):37-40.

12. Kaiser JM, Collares MVM, Pinto RA, Oliveira $\mathrm{ACP}$, Portinho $\mathrm{CP}$, Viaro MS. Tratamento cirúrgico de macroglossia na síndrome de BeckwithWiedemann: relato de caso. Arq Catarin Med. 2007; 36(supl1):138-9.

13. Matsune K, Miyoshi K, Kosaki R, Ohashi H, Maeda $\mathrm{T}$. Taste after reduction of the tongue in Beckwith-Wiedemann syndrome. $\mathrm{Br} \mathrm{J}$ Oral Maxillofac Surg. 2006; 44(1):49-51.

14. D'Agostino L, Rode R, Cunha KN. Síndrome de Beckwith-Wiedmann relato de caso com acompanhamento seqüencial de dezesseis anos. In: Coleção Sociedade Brasileira de
Fonoaudiologia. Atualização em voz, linguagem, audição e motricidade oral. São Paulo: Frôntis; 1999. p.395-404.

15. Mühle GC, Limongi SCO. Síndrome de BeckwithWieedmann: proposta de atuação fonoaudiológica precoce e relato de caso. Rev Soc Bras Fonoaudiol. 2001; 6(1):52-8.

16. Marchesan IQ. Protocolo de avaliação miofuncional orofacial. In: Krakauer LH, Di Francesco RC, Marchesan IQ. Respiração oral: abordagem interdisciplinar. São José dos Campos: Pulso; 2003. p.61-78.

17. Hernandez AM. Atuação fonoaudiológica com recém-nascidos e lactentes disfágicos. In: Hernandez AM, Marchesan IQ. Alterações fonoaudiológicas no ambiente hospitalar. Rio de Janeiro: Revinter; 2001. p.1-37.

18. Vieira JCF, Carneiro FM, Assêncio-Ferreira VJ. Alteração de deglutição em um caso de glicogenose. Rev. CEFAC. 2004; 6(1):34-9.

19. Castillo-Morales R. Terapia de regulação orofacial. São Paulo: Memnon; 1999.

20. Andrade FV, Andrade DV, Araújo AS, Ribeiro ACC, Deccax LDG, Nemr K. Alterações estruturais de órgãos fonoarticulatórios e más oclusões dentárias em respiradores orais de 6 a 10 anos. Rev. CEFAC. 2005; 7(3):318-25.

21. Dell'Aringa AR, Juares AJC, Melo C, Nardi JC, Kobari K, Perches Filho RM. Análise histopatológica de produtos de adenotonsilectomia de janeiro de 2001 a maio de 2003. Rev Bras Otorrinolaringol. 2005; 71(1):18-22.

22. Wang J, Goodger NM, Pogrel MA. The role of tongue reduction. Oral Surg Oral Med Oral Pathol Oral Radiol Endod. 2003; 95(3):269-73.

23. Picinato MNC, Trawtzki LVV, Anselmo-Lima WT. Proposta de atuação fonoaudiológica em um caso de macroglossia. Rev Fono Atual 2003; 6(25):57-63.

24. Bezerril DD, Barreto J, Macari S, Felício CM. A língua: características morfológicas normais e alterações. JBP Rev Ibero-Am Odontopediatr Odontol Bebê. 2005; 8(43):264-70.

DOI: 10.1590/S1516-18462010005000013

RECEBIDO EM: 14/06/2009

ACEITO EM: 10/11/2009

Endereço para correspondência:

Bárbara de Lavra-Pinto

Av. Venâncio Aires, 70 / 401

Porto Alegre - RS

CEP: 90040-190

E-mail: barbaradlp@ig.com.br 